\title{
¿Cómo surgen las ideas? Algunas sugerencias para estimular la generación de proyectos originales en ecología
}

\author{
Alejandro G. Farji-Brener \\ Laboratorio de Investigaciones en Hormigas (LIHO) - INIBIOMA - CONICET y Universidad Nacional del Comahue, Centro \\ Regional Universitario Bariloche. San Carlos de Bariloche, Río Negro, Argentina.
}

\begin{abstract}
RESUMEN. Los investigadores en ecología pretendemos desarrollar proyectos basados en ideas novedosas pero, como todos los seres humanos, tenemos una capacidad de creatividad limitada. Por ende, es común que durante nuestra vida académica se nos ocurran sólo unas pocas ideas realmente originales. Entonces, conocer cómo surgen las ideas se torna vital. Si estamos al inicio de la vida académica, nos facilitará el desarrollo de los primeros proyectos independientes, y si estamos hacia el final, puede alargar la etapa creativa de la vida profesional. En este ensayo, primero describiré algunas sugerencias para estimular la generación de proyectos originales en ecología y detallaré el contexto en el cual se gestaron. Finalmente, propondré un marco conceptual integrador cuyo uso puede estimular el surgimiento de ideas originales. En base a experiencias personales que he detallado en el texto, identifico tres aspectos que pueden estimular la creatividad: el inconformismo con las ideas dominantes, la existencia de contratiempos y la atención por lo atípico. Niveles extremos de estos aspectos son contraproducentes. Niveles mínimos de inconformismo, contratiempos o interés por lo atípico atentan contra la creatividad porque estimulan la sumisión intelectual a los paradigmas dominantes, fomentan la comodidad y promueven ceguera antes los detalles por fuera de la norma. Pero niveles muy altos también limitan la creatividad porque estimulan la crítica sin fundamentos, generan estrés permanente y estimulan el detallismo excesivo, descontextualizando un fenómeno de su entorno. En consecuencia, propongo que la generación de ideas originales se ve maximizada con niveles intermedios de inconformismo, contratiempos y atención a lo atípico. Para estimular proyectos en ecología basados en ideas originales sugiero poseer algún nivel de inconformismo sobre las ideas dominantes, considerar a los pequeños contratiempos como oportunidades y prestar atención a lo atípico sin desatender el contexto de donde provienen.
\end{abstract}

[Palabras clave: atípico, contratiempos, ideas, inconformismo, proyectos en ecología]

Aвstract. How do ideas arise? Some suggestions to stimulate the generation of original projects in ecology. Researchers in ecology try to develop projects based on novel ideas, but, as good human beings we are, we have a limited capacity for creativity. Therefore, it is usual for us, during our academic life, to generate only few really original ideas. Knowing how original ideas arise becomes vital. If we are at the beginning of our academic life, it will facilitate the development of our first independent projects; and if we are at the end of our career, it may lengthen the creative stage of our professional life. In this essay, I will first describe some suggestions to stimulate the generation of original projects in ecology, detailing the context where they were conceived. Finally, I will propose an integrative conceptual framework whose use may favor the occurrence of original ideas. Based on detailed personal experiences in the text, I identify three key aspects that may stimulate the generation of creativity: nonconformity with dominant ideas, the existence of setbacks and attention to the atypical. Extreme levels of these aspects are counterproductive. Minimal dosages of nonconformity, setbacks, or attention to the outliers undermine creativity because they stimulate conformity, comfort, and focus only on the rule. But too high levels also limit the generation of original ideas because it limit an integrative vision, causes exasperation and prevents us from seeing the phenomenon context. Consequently, I consider that the generation of original ideas is maximized with intermediate levels of nonconformity, setbacks and attention to the atypical. To stimulate ecology projects based on original ideas, I suggest having some level of nonconformity about the dominant ideas, considering small setbacks as opportunities and paying attention to the atypical without neglecting the context where they come from.

[Keywords: atypical, ecological projects, nonconformity, setbacks] 


\section{¿Cuántas veces podremos decir ¡EUREKA!?}

Los investigadores nos enfrentamos a una paradoja: pretendemos que nuestros proyectos de investigación sean novedosos, pero - como la gran mayoría de los seres humanos - tenemos una capacidad de creatividad limitada. En consecuencia, es probable que durante nuestra vida académica se nos ocurran sólo unas pocas ideas realmente originales. La ecología está llena de ejemplos de este fenómeno: reconocemos a ciertos investigadores por haber aportado una o dos ideas originales, no por haber propuesto veinte o treinta. Por mencionar a algunos, recordamos a D. Janzen (1970) y a J. H. Connell (1971) principalmente por sus modelos para explicar la diversidad integrando factores denso-dependientes y enemigos naturales, a E. L. Charnov (1976) por fundar las bases de la teoría de forrajeo óptimo, a E. O. Wilson (1975) por proponer la teoría de la sociobiología, a J. P. Grime (1977) por su triángulo que tipificaba las estrategias de la vegetación, a E. H. Rapoport (1982) por su trabajo sobre la variación en los rangos geográficos de las especies con la latitud, regla que posee su apellido (Rapoport 1982), a Coley (1983) por la hipótesis de la disponibilidad de recursos para explicar las variaciones intraespecíficas en las tasas de herbivoría, a R. Dirzo por estudiar los efectos de la defaunación contemporánea sobre la diversidad vegetal (Dirzo and Miranda 1991), a S. Díaz por su propuesta de diversidad funcional y su papel sobre la dinámica de los ecosistemas (Díaz and Cabido 2001) y a S. P. Hubbell (2001) por su teoría neutral de la biodiversidad, entre otros. El lector puede considerar este fenómeno como una buena o una mala noticia. Si usted está deprimido porque aún no se le han ocurrido grandes ideas, la buena noticia es que todavía tiene probabilidades de que la musa inspiradora lo visite. Y si usted está feliz porque siente que ya es autor de alguna buena idea, la mala noticia es que, probablemente, su imaginación esté llegando al límite. En ambos casos, conocer los mecanismos a través de los cuales surgen ideas originales se torna vital. Si estamos en la primavera de nuestra vida académica, nos facilitará el desarrollo de nuestros primeros proyectos independientes. Y si estamos en el otoño de nuestra carrera, puede alargar la etapa creativa de nuestra vida profesional.

La creación de ideas novedosas es un tema que excede a los interesados en realizar proyectos originales en ecología. Publicistas, artistas, empresarios, arquitectos y economistas (entre otras profesiones o actividades) también están interesados en comprender cómo estimular la creatividad. Sin embargo, pese a la enorme cantidad de información y conceptualización sobre esta temática, las sugerencias relacionadas con nuestra profesión son, en general, poco específicas; proponen que el surgimiento de las ideas depende de la experiencia, el azar, del conocimiento y de los estímulos externos (Elisondo et al. 2012; Elisondo 2016). También se plantea que las dos principales fuentes de inspiración para un ecólogo son la observación de la naturaleza y la lectura (Lawton 1996; Underwood et al. 2000), pero, hasta donde sé, no se ha profundizado en los mecanismos que fomentan el desarrollo de proyectos de investigación creativos en ecología.

En este ensayo plantearé algunas sugerencias para estimular la generación de proyectos originales en ecología y detallaré el contexto en el que se gestaron. Este objetivo posee dos propósitos. Por un lado, lograr que los lectores se identifiquen con las situaciones que describiré a continuación, y que al conocer 'la cocina' de cómo se genera un proyecto, comprendan que el proceso de investigación científica está plagado de dudas, errores, improvisaciones y contratiempos. Y por otra parte, pretendo plantear un marco conceptual integrador que se pueda emplear para estimular el desarrollo de proyectos de investigación novedosos. Sin embargo, antes de desarrollar mis ideas vale la pena realizar algunas aclaraciones. Primero, en este ensayo no propongo que la originalidad es el único o el más importante atributo que debe tener un proyecto de investigación para ser valioso. Muchos proyectos de investigación, independientemente de su originalidad, producen información de base o desarrollan propuestas metodológicas que generan conocimiento útil y necesario. Segundo, pese a que yo me voy a enfocar en las experiencias individuales debido a que la creatividad es una cualidad personal (Marone and González del Solar 2007), el entorno también puede afectar la imaginación. Por ejemplo, encontrar una pregunta creativa que no ha sido respondida en un sistema muy estudiado puede ser más difícil que encontrarla en un sistema menos estudiado. Asimismo, un investigador con una formación más diversa y heterodoxa (i.e., que incluya otras disciplinas tanto científicas como artísticas) puede tener más elementos 
para desarrollar ideas novedosas. Finalmente, la dinámica del grupo de investigación, las características de los directores, el intercambio con colegas y el contexto histórico, entre otros, también influyen sobre la capacidad creativa (Elisondo et al. 2012; Elisondo 2016). Aclarados estos conceptos, desarrollaré entonces tres aspectos que, en mi experiencia personal, fueron determinantes para poder decir ¡Eureka!

\section{Nadar contra la corriente}

En general, las primeras experiencias de aprendizaje se logran a través de la repetición de elementos conocidos, lo cual puede atentar contra la creatividad. Por ejemplo, al aprender un instrumento musical, es casi inevitable aprender a tocar canciones existentes antes de componer temas propios. Algo similar ocurre en ciencia. La primera experiencia de investigación generalmente carece de originalidad porque se tiene la tendencia a describir patrones, repetir ideas ajenas o poner a prueba hipótesis conocidas. Yo no soy la excepción. Mi primer proyecto fue el de mi tesis de licenciatura, en la que pretendía describir la dieta de dos especies de hormigas cortadoras en un contexto de potencial competencia interespecífica. En ese momento (1985), el papel de la competencia en estructurar comunidades era un tópico dominante en ecología (Tilman 1982), y las hormigas cortadoras eran famosas básicamente por su gran capacidad defoliadora (Figura 1A, izquierda). Bajo la influencia de lo popular y lo conocido, no se me ocurrió mejor idea que comparar la dieta de dos especies de hormigas cortadoras de hojas para determinar si diferencias en sus estrategias forrajeras reducían su nivel de competencia, facilitando así su coexistencia. La tesis, y el artículo que surgió de ella (Farji-Brener and Protomastro 1992), fue una gota de agua más en el océano. Desde su aparición en 1992 hasta la fecha, ha acumulado sólo alrededor de 16 citas y estimo - más allá de su valor intrínseco de describir el comportamiento de ciertas especies en un sitio
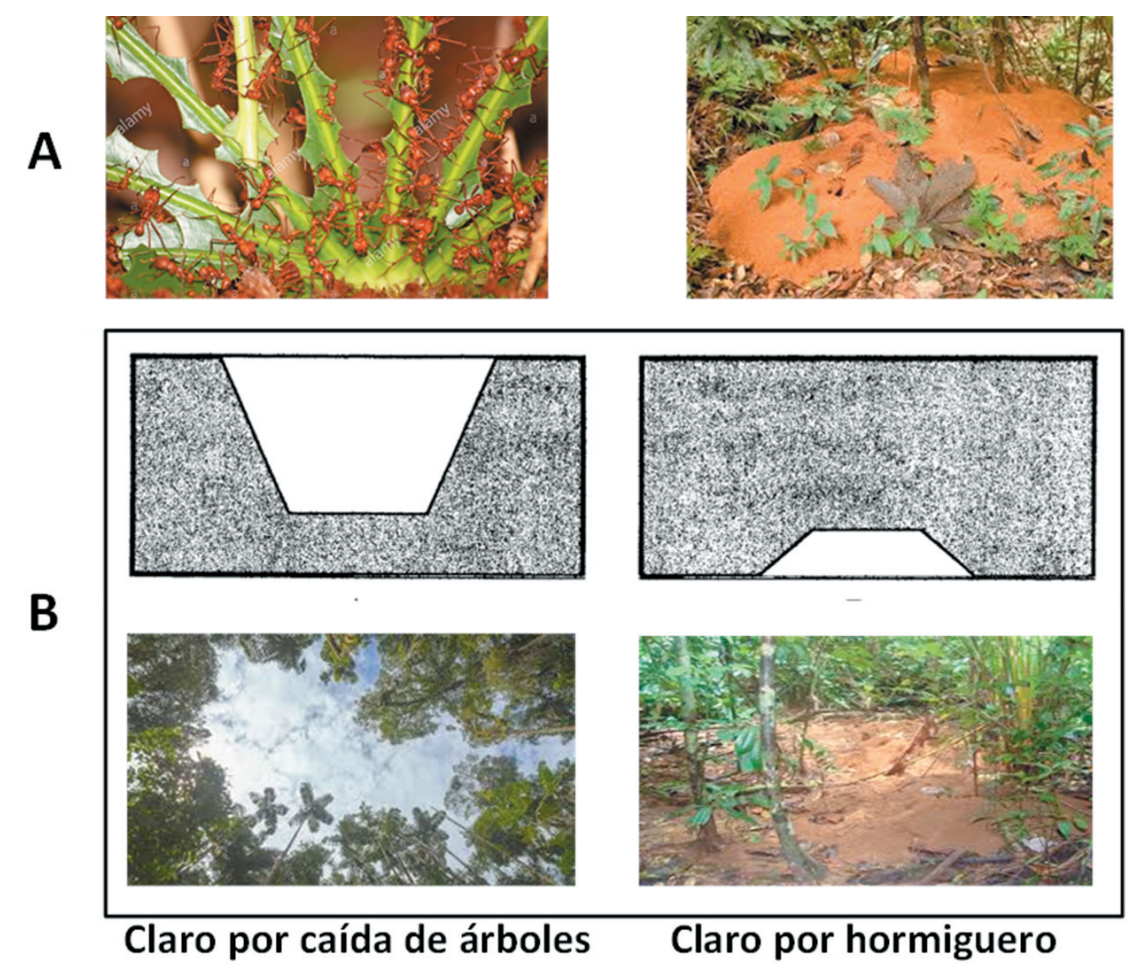

Figura 1. A) El efecto negativo de las hormigas cortadoras de hojas sobre las plantas (A, derecha) era mucho más conocido y estudiado que su potencial efecto positivo sobre la vegetación a través de los cambios en el suelo generado por sus hormigueros (A, izquierda). B) 'Sofisticado' esquema que resume el marco conceptual propuesto en Farji-Brener and Illes (2000), en el cual se proponía a los hormigueros como generadores de disturbios desde abajo hacia arriba (bottomup disturbances), en contraposición a las perturbaciones generadas por las caídas de árboles (top-down disturbances).

Figure 1. The negative effect of leaf-cutting ants on plants (A, right) was much better known and studied than their potential positive effect on vegetation through changes in the soil generated by their nests (A, left). B) 'Sophisticated' scheme summarizing the conceptual framework proposed by Farji-Brener and Illes (2000), in which ant nests were proposed as generators of bottom-up disturbances, as opposed to those generated by the tree falls (top-down disturbances). 
específico - que no aportó grandes novedades ni a la teoría de la competencia ni a la historia natural de las especies estudiadas.

Pese a la obtención de mi título de Licenciado en Biología, me quedé con el sabor amargo que deja la repetición de ideas ajenas. Aprendí mucho sobre mi organismo modelo y para mis futuros proyectos me propuse intentar ser un poco más innovador. Pero al acudir a la literatura existente para incentivar $\mathrm{mi}$ imaginación, la cantidad de referencias sobre el efecto negativo de las hormigas cortadoras sobre la vegetación era abrumadora. El nivel de información sobre su status como plaga era muy superior a cualquier otro tipo de aspecto de su historia natural o a cualquier otro efecto potencial sobre las plantas. Y entonces sucedió. La musa inspiradora acudió a mi puerta. Si todo el mundo se había centrado en estudiar los aspectos 'negativos' de las hormigas cortadoras de hojas, ¿por qué no enfocarse en los aspectos 'positivos'? Pese a que esta actitud podría ser catalogada como el típico comportamiento de llevar la contra, me propuse indagar sobre estos potenciales aspectos positivos. Y para mi sorpresa, encontré una temática prometedora casi inexplorada. En mis tesis de maestría y de doctorado, y durante mis estudios post doctorales me dediqué principalmente a examinar cómo las hormigas cortadoras de hojas beneficiaban a las plantas a través de las modificaciones físicas y químicas en el suelo alrededor de sus hormigueros (Figura 1A, derecha). Eso generó varios artículos sobre este tópico (e.g., Farji-Brener and Silva 1995a,b; Farji-Brener and Ghermandi 2000; Farji-Brener and Medina 2000), los cuales me permitieron proponer a los hormigueros como disturbios en un marco conceptual original (Farji-Brener and Illes 2000) (Figura 1B). A diferencia de aquel ignoto manuscrito sobre dieta y competencia, la idea plasmada en ese artículo tuvo un buen impacto en la comunidad científica, y estimuló una gran cantidad de estudios de otros investigadores. En resumen, nadar un poco contra la corriente me ayudó a visualizar temáticas poco exploradas y, en consecuencia, a desarrollar proyectos un poco más imaginativos que tuvieron un impacto mayor.

\section{Considerar los contratiempos como oportunidades}

Durante muchos años he participado como profesor en cursos intensivos de campo sobre ecología tropical, en los que, como parte de la dinámica, debía coordinar un pequeño proyecto de investigación con un grupo reducido de estudiantes. Ese año tenía una idea en la cabeza que había surgido de un proyecto anterior. Habíamos descubierto que las hormigas que cargaban hojas extragrandes iban más lentamente y retrasaban a sus compañeras que iban detrás, de la misma manera que un gran camión retrasa a los autos en una ruta. Pero lo más interesante era que la proporción de estas hormigas que llevaban cargas extra-grandes era muy baja cuando el flujo de hormigas en los senderos era alto, pero muy alta cuando el flujo de hormigas era bajo (Farji-Brener et al. 2011). Ese patrón sugería una regla de tránsito común en las sociedades humanas: para evitar retrasos y embotellamientos, los transportes con cargas pesadas estaban restringidos a los momentos de baja circulación. Probar esta hipótesis requería de más experimentación, y me propuse desarrollar ese proyecto para el próximo curso. Al año siguiente planteé la idea a mi grupo de estudiantes, quienes la aceptaron con entusiasmo. Íbamos a ofrecer fragmentos de tamaño normal y extra-grandes en senderos con niveles de flujo alto y bajo. Si existía la regla de restricción de tránsito para cargas pesadas, esperábamos que las hormigas eligieran cargar los fragmentos extra-grandes principalmente en condiciones de flujo bajo. Todos estábamos contentos con el proyecto y animados a salir al campo a realizar los ofrecimientos y recolectar los datos. Pero como el curso se desarrollaba en el bosque tropical húmedo, sucedió lo predecible: llovió. Y llovió y llovió y llovió. Y las hormigas no salen con lluvia.

Aún recuerdo ese momento, vueltos del campo completamente empapados y desilusionados por no haber visto ni una sola hormiga, reunidos en un aula pensando un plan alternativo. Estábamos empeñados en trabajar con hormigas, pero el nuevo plan no podía depender de su actividad. Mientras afuera llovía torrencialmente, dentro del aula ocurría otra tormenta, la famosa 'tormenta de cerebros'. Todos pensábamos qué cosas podíamos medir de las hormigas sin necesidad de su presencia, cuando entonces sucedió. La musa inspiradora acudió a nuestra aula. Ahí estaban los senderos de forrajeo, por donde las hormigas se dirigen a la vegetación que cortan y por donde regresan al nido con alimento. Dado que las hormigas los mantienen libres de vegetación y de hojarasca, estos 
senderos son muy visibles aunque no haya hormigas transitando por él. Si se considera que la mayoría de estos senderos poseen bifurcaciones construidas para acceder a un nuevo parche de alimentación, y que estas bifurcaciones se pueden construir con diferentes ángulos respecto al sendero troncal, se nos ocurrió preguntarnos si dichos ángulos variaban dependiendo de la importancia relativa de reducir el costo de mantenimiento de la bifurcación o la distancia de viaje. Lo bonito era que ambos costos no pueden reducirse simultáneamente. Por ejemplo, si se pretende unir un recurso con el sendero troncal construyendo un nuevo tramo, diseñarlo a $90^{\circ}$ es la mejor opción para reducir costos de mantenimiento porque determina que la bifurcación tenga la mínima longitud posible, pero es la peor opción para reducir distancias (i.e., ya que es el atajo más largo). Por otra parte, las bifurcaciones con ángulos menores a $90^{\circ}$ son más eficientes para reducir distancias (i.e., son atajos más cortos), pero determinan la construcción de un nuevo tramo más largo, incrementando los costos de mantenimiento (Figura 2). Esta idea novedosa tenía predicciones muy claras: nidos en ambientes abiertos, en donde el costo de mantenimiento era casi nulo al no haber hojarasca cayendo del dosel pero que reciben el efecto negativo directo del sol y la lluvia, debían presentar bifurcaciones con ángulos más agudos (o sea, priorizar la reducción del costo de viaje). Por otra parte, los nidos dentro del bosque debían presentar bifurcaciones con ángulos cercanos a los $90^{\circ}$, ya que allí el costo de viaje no es tan severo porque los senderos están protegidos del efecto directo del sol y la lluvia, pero poseen costos de mantenimiento elevados al existir una caída constante de hojarasca. La medición era increíblemente sencilla: sólo había que salir bajo la lluvia a medir ángulos de bifurcaciones en la mayor cantidad de senderos y nidos posibles, sin importar que las hormigas no estuvieran allí. En otras palabras, íbamos a estudiar el comportamiento de un organismo a través de sus construcciones, lo que comúnmente se denomina 'fenotipo

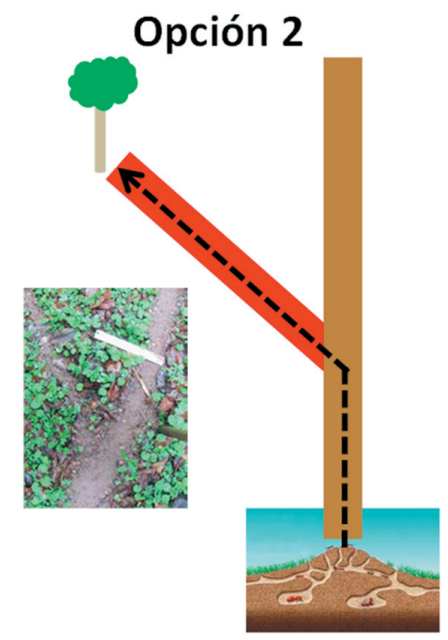

Figura 2. Ejemplo de cómo realizamos un estudio sobre hormigas sin su presencia. Cuando las hormigas deben construir una bifurcación para unir el sendero de forrajeo troncal con un nuevo recurso, la pueden realizar a diferentes ángulos. La opción 1 muestra el ángulo que reduce los costos de construcción y mantenimiento $\left(90^{\circ}\right)$ - ya que determina la menor longitud posible del nuevo tramo-, pero que es la peor opción para acortar distancias. La opción 2 muestra que los ángulos $<90^{\circ}$ son mejores para acortar distancias, pero representan la peor opción para reducir costos de construcción y mantenimiento porque la longitud del nuevo tramo aumenta. Nosotros predijimos que los nidos dentro del bosque, donde los costos de mantenimiento son altos por la caída constante de hojarasca, pero los costos de viaje son menores por estar protegidos del impacto directo del sol y de la lluvia, debían tener ángulos cercanos a $90^{\circ}$. Por el contrario, los nidos en áreas abiertas, donde los costos de mantenimiento son casi nulos por la ausencia de hojarasca, pero los costos de viaje son altos por la incidencia directa del sol y la lluvia, debían tener ángulos de bifurcación $<90^{\circ}$ (Farji-Brener et al. 2015).

Figure 2. Example of how we carried out a study on ants without their presence. When ants must build a trail bifurcation to connect the central trail with a new resource, it can be done at different angles. Option 1 shows the angle that reduces construction and maintenance costs $\left(90^{\circ}\right)$ since it determines the shortest possible length of the new section, but it is the worst option to shorten distances. Option 2 shows that angles $<90^{\circ}$ are better for shortening distances, but the worst option for reducing construction and maintenance costs. We predicted that nests within the forest, where maintenance costs are high due to the constant fall of leaf litter, but with the lower travel costs due to being protected from the direct impact of rain and sun, should have angles close to $90^{\circ}$. Conversely, nests in open areas, where maintenance costs are reduced due to the absence of leaf-litter, but travel costs are high due to direct incidence of sun and rain, should have bifurcation angles $<90^{\circ}$ (Farji-Brener et al. 2015). 
extendido' (Dawkins 1982). Siendo sincero, la idea nos parecía tan descabellada como interesante: pese a lo simple de su lógica, nadie la había planteado antes. Fuimos al campo con mínimas expectativas de que se cumpliesen nuestras predicciones, pero los resultados fueron asombrosos. Finalmente, ese proyecto originó una publicación en una reconocida revista de ecología (Farji-Brener et al. 2015). Pero lo más alentador fue el comentario de uno de los revisores, quien dijo textualmente: "Ojalá esta idea se me hubiese ocurrido a mí". Fue cuando pensé que deberíamos haber puesto a la lluvia como coautora, porque la idea surgió, en parte, gracias a ella. Este suceso me enseñó que, metafóricamente hablando, un contratiempo puede lograr que nos apartemos un poco del sendero principal que teníamos planificado, descubriendo bifurcaciones novedosas hacia destinos más originales.

\section{Aprovechar el contraste de lo atípico}

Ante lo atípico uno puede voltear la cabeza y quedarse con la norma, o aprovechar la oportunidad de lo diferente para indagar explicaciones novedosas y estudiar mecanismos inexplorados. ¿Quién hubiese pensado que hay plantas sin clorofila, frutos adaptados a fauna ya extinta, o sapos cantores sordos? Sin embargo, hay plantas con flores que no realizan fotosíntesis (Kuijt 1969), árboles cuyos frutos presentan adaptaciones para ser dispersados por fauna desaparecida hace 10000 años (Janzen and Martin 1982) y sapos con el sistema auditivo disfuncional (Goutte et al. 2017). El estudio de estos aspectos aparentemente fuera de la norma puede estimular ideas novedosas e hipótesis creativas sobre temas descuidados por la mayoría de los investigadores.

Todos los grupos de organismos presentan aspectos que parecen contrastantes o atípicos. Las hormigas cortadoras no son la excepción. Estos insectos cortan hojas con las cuales cultivan un hongo mutualista, que es el principal alimento de las larvas y las pupas. Pero el proceso de cultivo y crecimiento del hongo genera residuos orgánicos en cantidades considerables (las colonias de estas hormigas pueden alcanzar millones de individuos), residuos que son una fuente potencial de patógenos para el hongo que cultivan y para las propias hormigas (Currie 1999; Reynolds and Currie 2004). Sin embargo, y a pesar de las enormes similitudes entre las especies de hormigas cortadoras de hojas en cuanto a su historia natural, algunas depositan su basura en cámaras subterráneas y otras las acumulan en montículos sobre la superficie del suelo. Esta evolución de estrategias tan contrastantes en especies tan similares me estimuló a formular hipótesis que hasta el momento no se habían propuesto explícitamente. Propusimos que este comportamiento contrastante dependía de las condiciones del ambiente: en sistemas cuyo clima favorecía la propagación de patógenos (i.e., selvas tropicales y subtropicales húmedas) debía ser más higiénico enterrar los residuos, pero en ambientes donde la probabilidad de proliferación de patógenos era menor (i.e., desiertos) se podía 'ahorrar' el esfuerzo de construir cámaras subterráneas y depositar la basura en montículos externos sin tanto riesgo de contagio.

Para poner a prueba esta idea, desarrollamos experimentos en los que analizamos las condiciones térmicas de proliferación de los patógenos, usamos información bibliográfica y realizamos análisis filogenéticos. Finalmente, encontramos que el comportamiento de enterrar o acumular basura en la superficie estaba en parte determinado por la filogenia, pero también por la ecología. Como predijimos, las especies de cortadoras que habitaban ambientes más desérticos depositaban la basura en montículos externos, y las especies que habitaban en ambientes más húmedos generalmente enterraban sus desechos (FarjiBrener et al. 2016). En resumen, las ideas novedosas aparecieron porque intentamos explicar un aspecto contrastante de la historia natural de las hormigas cortadoras. Es muy probable que todos los grupos de organismos presenten contrastes o aspectos atípicos que la mayoría de los investigadores ignorará en pos de estudiar los patrones más generales. Sin embargo, prestar atención a los aspectos contrastantes de la historia natural de un organismo o a las situaciones que se salen por fuera de la norma puede incentivar ideas novedosas y proyectos creativos.

\section{Integrando las sugerencias en un marco conceptual común}

La creatividad es un requisito importante de las ciencias, ya que los científicos muchas veces requieren la activación de procesos vinculados a la producción de nuevos conocimientos (Elisondo 2016). En mi experiencia personal, detecté que ser inconformista, sufrir contratiempos y prestar atención a lo atípico fueron comportamientos que me estimularon a desarrollar ideas originales. Algunos de 
estos aspectos ya han sido señalados como promotores de la creatividad. Por ejemplo, la capacidad de confrontar obstáculos se ha vinculado estrechamente con las posibilidades de desarrollar procesos creativos. La escasez de recursos puede afrontarse agregando más creatividad a los procesos, generando los recursos necesarios para desarrollar ideas y resolver problemas al transformar las dificultades en posibilidades (Elisondo et al. 2013; Elisondo 2016). Por otra parte, el investigador creativo no puede ser intelectualmente perezoso; debe tener espíritu crítico, ser curioso, inconformista y un poco escéptico (Marone and González del Solar 2007). En este sentido, los contratiempos y el espíritu crítico ya fueron reconocidos como motores de la creatividad, aunque el interés por lo atípico ha despertado menos interés.

Sin embargo, los extremos pueden atentar contra la imaginación. Mi propuesta es que la máxima creatividad se logra con niveles intermedios de inconformismo, contratiempos y atención a lo atípico. No criticar nunca los paradigmas dominantes desestimula la generación de ideas novedosas porque incita la sumisión intelectual. Pero estar todo el tiempo llevando la contra (i.e., la crítica sin fundamento sólido, o como deporte per se) también puede sesgar nuestra objetividad y limitar nuestra creatividad. En el mismo sentido, la falta absoluta de contratiempos ubica al investigador en una zona de confort que normalmente limita la imaginación. Si todo sale como lo planificamos, la imaginación se adormece bajo un manto de comodidad. Pero la presencia permanente de contratiempos puede generar un nivel de estrés que también obstruya la generación de ideas novedosas. Finalmente, ignorar aspectos atípicos desalienta la ocurrencia de explicaciones novedosas, pero descontextualizar esas anomalías de su entorno estimula un detallismo excesivo que también limita la imaginación. En resumen, ambos extremos parecen ser nocivos para la creatividad, ya que restringen el surgimiento de ideas originales. En consecuencia, la generación de ideas originales se maximizará con niveles intermedios de inconformismo, contratiempos y atención por lo atípico (Figura 3). Cuáles son los niveles 'intermedios' dependerá de la idiosincrasia de cada investigador, de las
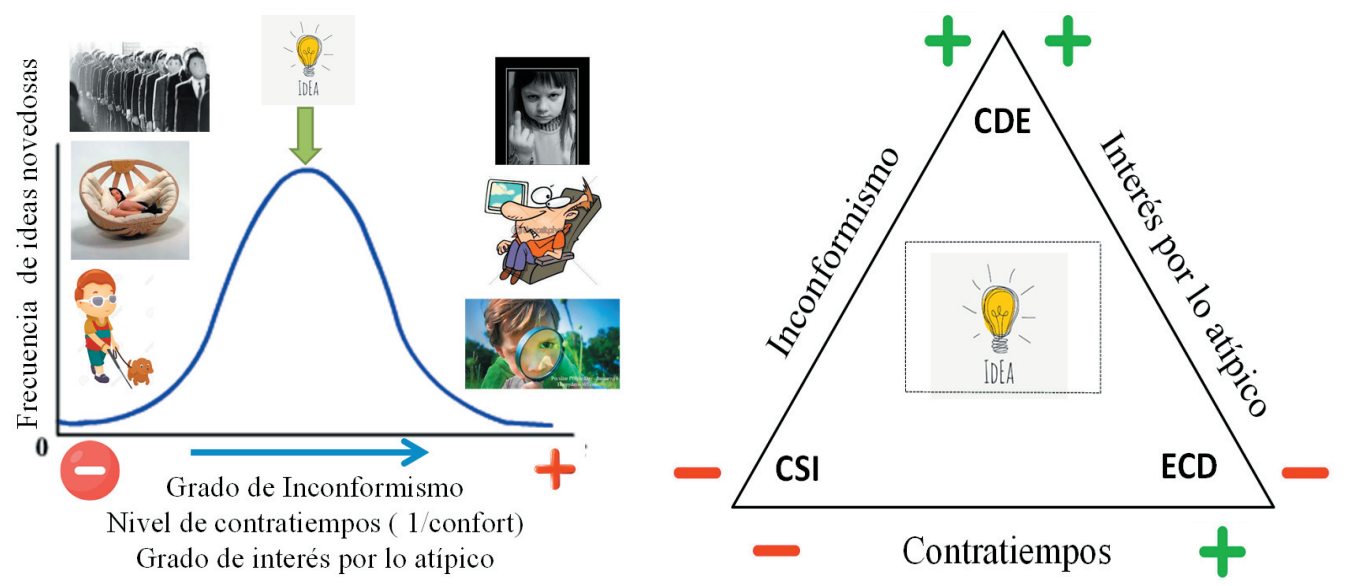

Figura 3. Marco conceptual bajo el cual se propone que la generación de ideas originales se maximiza con niveles intermedios de inconformismo, contratiempos e interés por lo atípico. Niveles mínimos de inconformismo, contratiempos o interés por lo atípico atentan contra la creatividad porque estimulan la sumisión intelectual a los paradigmas dominantes, fomentan la comodidad y promueven ceguera ante los detalles que se salen de la norma. Pero niveles demasiado altos también limitan la generación de ideas originales porque estimulan la crítica sin fundamentos, generan estrés permanente (dificultando la imaginación) y estimulan el detallismo excesivo, descontextualizando un fenómeno de su entorno. En el esquema de la derecha, los extremos del triángulo ilustran situaciones donde se fomentan las Críticas y el Detallismo Excesivo (CDE), la Comodidad y la Sumisión Intelectual (CSI), y el Estrés y la Ceguera a los Detalles (ECD), todas situaciones que desalientan la generación de ideas originales.

Figure 3. Conceptual framework in where is proposed that the generation of original ideas is maximized with intermediate levels of nonconformity, setbacks and interest in the atypical. Minimal levels of non-conformity, setbacks or interest in the atypical undermine creativity because they encourage intellectual submission to dominant paradigms, foster comfort and promote blindness to details that are out of the norm. But too high levels also limit the generation of original ideas because they deprive us of an integrative vision, cause exasperation and prevent us from seeing the complete panorama of a phenomenon. In the diagram on the right, the ends of the triangle illustrate situations that promote Criticism and Excessive Detailing (CDE), Comfort and Intellectual Submission (CSI), and Stress and Detail Blindness (ECD), all situations that discourage the generation of original ideas. 
circunstancias y del sistema en estudio; todos aspectos que pueden variar entre proyectos y con el paso del tiempo. En mi experiencia, el encuentro de este 'punto medio' fue estimulado por un mentor que me enseñó a confiar más en los datos que en los libros, me entrenó en el arte de la observación de los detalles sin desatender el contexto y me enseñó a aprovechar los contratiempos que ocurren en el campo, donde las cosas nunca suceden como se planifican en la oficina. No es el objetivo de este ensayo dar recetas sobre cómo encontrar ese punto medio que estimula la creatividad; espero que cada lector pueda hallarlo por sí mismo. Pero a los que deseen que la musa inspiradora acuda a su puerta, les puedo sugerir que se permitan dudar de las ideas dominantes, que consideren a los pequeños contratiempos como oportunidades, y que presten atención a lo atípico sin desatender su contexto.

Agradecimientos. Los comentarios de dos revisores anónimos y el editor temático mejoraron notablemente la primera versión de este manuscrito.

\section{REFERENCIAS}

Charnov, E. L. 1976. Optimal foraging: The marginal value theorem Theoretical Population Biology 9:129-136. https: //doi.org/10.1016/0040-5809(76)90040-X.

Coley, P. D. 1983. Herbivory and defensive characteristics of tree species in a lowland tropical forest. Ecological Monographs 53:209-234. https://doi.org/10.2307/1942495.

Connell, J. H. 1971. On the role of natural enemies in preventing competitive exclusion in some marine animals and in rain forest trees. Pp. 298-312 in den Boer, P. J. and G. R. Gradwell (eds.). Dynamics of Numbers in Populations. PUDOC, Wageningen, The Netherlands.

Currie, C. R., J. A. Scott, R. C. Summerbell, and D. Malloch. 1999. Fungus-growing ants use antibiotic-producing bacteria to control garden parasites. Nature 398:701-704. https://doi.org/10.1038/19519.

Dawkins, R. 1982. The extended phenotype (Vol. 8). Oxford: Oxford University Press.

Díaz, S., and M. Cabido. 2001. Vive la différence: plant functional diversity matters to ecosystem processes. Trends in Ecology and Evolution 16:646-655. https://doi.org/10.1016/S0169-5347(01)02283-2.

Dirzo, R., and A. Miranda. 1991. Altered patterns of herbivory and diversity in the forest understory: a case study of the possible consequences of contemporary defaunation. Pp. 273-287 in Plant-animal interactions: evolutionary ecology in tropical and temperate regions. Wiley, New York.

Elisondo, R. C. 2016. Creatividad y Ciencias Un estudio biográfico de científicos argentinos. Ciencia, Docencia y Tecnología 27(52):6.

Elisondo, R. C., D. S. Donolo, and M. CC. Rinaudo. 2012. Houssay, Leloir y Milstein: procesos creativos en las ciencias. Universidad Nacional de San Luis, Argentina. Fundamentos en Humanidades II(26):99-114.

Farji Brener, A. G., and J. Protomastro. 1992. Patrones forrajeros de dos especies simpátricas de hormigas cortadoras de hojas (Attini, Acromyrmex) en el Chaco seco. Ecotrópicos 5:32-43.

Farji Brener, A. G., and J. F. Silva. 1995a. Leaf-cutting ants and forest groves in a tropical parkland savanna of Venezuela: facilitated succession? Journal of Tropical Ecology 11:651-669. https://doi.org/10.1017/S0266467400009202.

Farji Brener, A. G., and J. F. Silva. 1995b. Leaf-cutting ant nests and soil fertility in a well-drained savanna in western Venezuela. Biotropica 27:250-253. https://doi.org/10.2307/2389001.

Farji-Brener, A. G., and A. Illes. 2000. Do leaf-cutting ant nests make 'bottom up' gaps in Neotropical rain forests? A critical review of the evidence. Ecology Letters 3:219-227. https://doi.org/10.1046/j.1461-0248.2000.00134.x.

Farji-Brener, A. G., and C. Medina. 2000. The importance of where to dump the refuse: seed banks and fine roots in nests of the leaf-cutting ants Atta cephalotes and Atta colombica. Biotropica 32:120-126. https://doi.org/10.1111/j.17447429.2000.tb00454.x.

Farji-Brener, A. G., and L. Ghermandi. 2000. The influence of nests of leaf-cutting ants on plant species diversity in road verges of northern Patagonia. Journal of Vegetation Science 11:453-460. https://doi.org/10.2307/3236638.

Farii-Brener, A. G., F. Chinchilla, S. Rikkin, A. M. Sánchez-Cuervo, E. Triana, V. Quiroga, and P. Giraldo. 2011. The "truck-driver" effect in leaf-cutting ants: how individual load influences the walking speed of nest-mates. Physiological Entomology 36:128-134. https://doi.org/10.1111/j.1365-3032.2010.00771.x.

Farji-Brener, A. G., F. Chinchilla, m. N. Umaña, M. E. Ocasio-Torres, A. Chauta-Mellizo, D. Acosta-Rojas, et al. 2015. Branching angles reflect a trade-off between reducing trail maintenance costs or travel distances in leaf-cutting ants. Ecology 96:510-517. https://doi.org/10.1890/14-0220.1.

Farji-Brener, A. G., L. Elizalde, H. Fernández-Marín, and S. Amador-Vargas. 2016. Social life and sanitary risks: evolutionary and current ecological conditions determine waste management in leaf-cutting ants. Proceedings of the Royal Society - Series B 283:20160625. https://doi.org/10.1098/rspb.2016.0625.

Goutte, S., M. J. Mason, J. Christensen-Dalsgaard, F. Montealegre-Z, B. D. Chivers, F. A. Sarria-S, et al. 2017. Evidence of auditory insensitivity to vocalization frequencies in two frogs. Scientific Reports 7:1-9. https://doi.org/10.1038/ s41598-017-12145-5.

Grime, J. P. 1977. Evidence for the existence of three primary strategies in plants and its relevance to ecological and evolutionary theory. American Naturalist 111:1169-1194. https://doi.org/10.1086/283244. 
Hubbell, S. P. 2001. The Unified Neutral Theory of Biodiversity and Biogeography. Princeton University Press.

Janzen, D. H. 1970. Herbivores and the number of tree species in tropical forests. Am Nat 104:501-528. https://doi.org/ $10.1086 / 282687$.

Janzen, D. H., and P. S. Martin. 1982. Neotropical anachronisms: the fruits the gomphotheres ate. Science 215:19-27. https://doi.org/10.1126/science.215.4528.19.

Kozbelt, A., R. A. Beghetto, and M. A. Runco. 2010. Theories of creativity. Pp. 20-46 in Kaufman, J. C. and R. J. Sternberg (eds.). Cambridge handbook of creativity. Chapter 2. Cambridge University Press. https://doi.org/10.1017/ CBO9780511763205.004

Kuijt, J. 1969. The biology of parasitic flowering plants. University of California Press, Berkeley. The biology of parasitic flowering plants. University of California Press, Berkeley.

Lawton, J. 1996. Patterns in ecology. Oikos 75:145-147. https://doi.org/10.2307/3546237.

Marone, L., and González del Solar, N. 2007. Crítica, creatividad y rigor: vértices de un triángulo culturalmente valioso. Interciencia 32:354-357.

Rapoport, E. H. 1982. Aerography: geographical strategies of species. Pergamon.

Reynolds, H. T., and C. R. Currie. 2004. Pathogenicity of Escovopsis weberi: the parasite of the attine ant-microbe symbiosis directly consumes the ant-cultivated fungus. Mycologia 96:955-959. https://doi.org/10.1080/ 15572536.2005 .11832895$.

Tilman, D. 1982. Resource competition and community structure. Princeton University Press. https://doi.org/10.1515/ 9780691209654.

Underwood, A. J., M. G. Chapman, and S. D. Connell. 2000. Observations in ecology: you can't make progress on processes without understanding the patterns. Journal of Experimental Marine Biology and Ecology 250:97-115. https://doi.org/10.1016/S0022-0981(00)00181-7.

Wilson, E. O. 1975. Sociobiology: The New Synthesis. Harvard University Press. Cambridge (MA). 